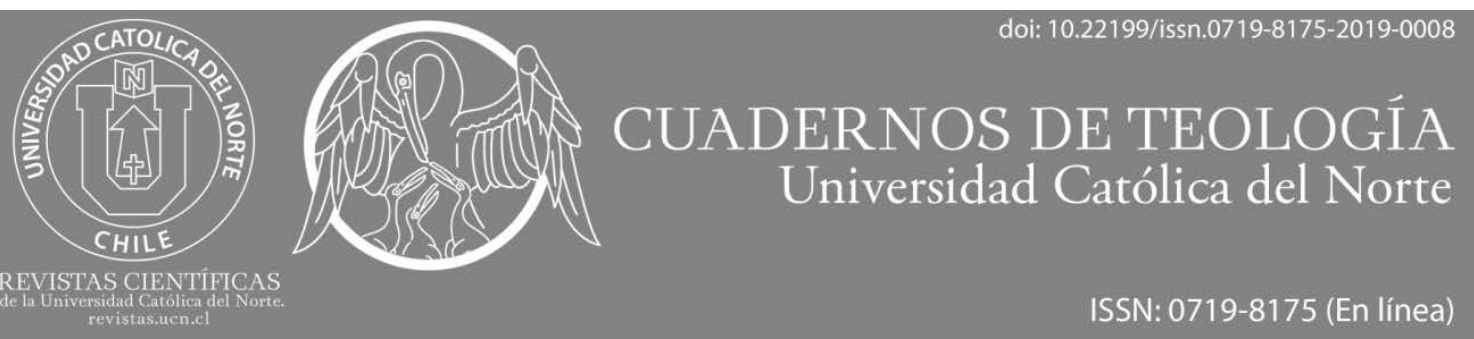

\title{
De la post verdad a la verdad
}

\section{From the post-truth to the truth}

\section{Christian Naranjo Navas* (D) https://orcid.org/0000-0003-1532-203X}

"Universidad Nacional de Chimborazo, Riobamba, Ecuador. Profesor. PhD. en Análisis Económico, U. Autónoma de Barcelona. paulnaranjo@outlook.com

(cc) BY

\section{Resumen:}

Se intenta visualizar los peligros de la era de la post verdad, mientras relativiza lo relativo para argumentar que los postulados de esta era son irracionales. La única forma de entender una estructura moral en la sociedad de la post verdad es a través de la imposición, de la persuasión o de la manipulación. En todos los casos, la moral queda relegada a lo subjetivo. Al final, se ha propuesto una argumentación basado en la ley de la no contradicción para entender que la verdad existe, que la verdad es el logos [^óyoc] absoluto, y este logos [^óyoc] es Dios.

\section{Palabras Clave: Mentira emotiva; Cristo; Moralidad; Post factual.}

\section{Abstract:}

An attempts to visualize the dangers of the post-truth era, while relativizing the relative to argue that the postulates of this era are irrational. The only way to understand a moral structure in a post-truth society is through imposition, persuasion or manipulation. In all cases, morality is relegated to the subjective. In the end, an argument based on the law of noncontradiction has been proposed to understand that truth exists, that truth is the absolute logos [^óyoc], and this logos [^óyoc] is God.

Keywords: Emotional lie; Christ; Morality; Post-factual 


\section{Introducción}

La post modernidad nos presenta un cristianismo que se construye como un nido entrelazado entre costumbres y mitos, entrelazado entre percepciones adaptables a las circunstancias, un cristianismo construido en una cultura lista para justificar todo uso y creencia que surja de los pueblos, de la "sabiduría ancestral". Es fácil divisar como la cultura occidental de la post modernidad se ha visto pronta a adaptarse, a acomodarse intuitivamente entre la verdad y la post verdad, según le convenga, desee o sienta. La era de la post verdad nos presenta una cultura occidental adaptable, cambiante, y un cristianismo cultural pronto a justificar todos estos cambios, pronto a construirse como una plataforma de aceptación a todo uso, a toda creencia, a toda adaptación cultural.

La era de la post verdad ha creado un espacio amplio de tolerancia de lo todo, con colectivos que han tomado al cristianismo como plataforma para justificar sus nuevas posiciones, en una suerte de actos de expiación: todo es justificable, todo es adaptable, todo es útil para justificarse. La era de la post verdad nos brinda una visión difusa de la realidad: la concepción de verdad ha pasado de ser un principio estático, pertinente a todo y a todos, a ser una idea dinámica, adaptable, casi una visión innecesaria. Al mismo tiempo, todo y nada es justificable, todo y nada es aceptado, depende de los interlocutores, depende de las circunstancias. Todo es relativo, de esta forma, no se puede afirmar nada porque la relatividad cultural solo acepta, mientras intenta, en ocasiones, justificarse con la adaptación de un Jesús post moderno.
De esta forma, este ensayo pretende visualizar los peligros de la era de la post verdad, intenta relativizar lo relativo, de forma que se pueda mostrar que la "lógica" de esta era no encuentra fundamento, sino solo una adaptación a cada circunstancia, y una aceptación creciente a todo uso cultural. Al mismo tiempo, el ensayo pretende recrear la justificación racional de la existencia de verdad, justificada en la existencia de Dios. Esto involucra la existencia de principios universales, no adaptables ni manipulables, encontrados al contrastar la verdad con la post verdad. Las pretensiones del ensayo se presentan como una colosal tarea, sin embargo, encontramos ánimo al revisar las obras de Zacharias (2000; 2008; 2012), Lennox (2007; 2011; 2015), Turek y Geisler (2010), Turek (2014), y Koukl $(2011 ; 2017)$, quienes han trabajado de forma sistemática en entender la verdad de forma racional, mientras exponen la irracionalidad de los postulados de la era de la post verdad.

\section{Post verdad y cultura}

En 2016, Oxford Dictionaries decidió escoger la palabra "post truth", como la palabra del año, es decir, la palabra de mayor uso y popularidad (Oxford University Press, 2017). La noticia no nos tomó por sorpresa, era de esperarse, pues su uso ha incrementado exponencialmente. La post verdad no se refiere necesariamente a un evento posterior a otro, la composición de palabras no tiene una percepción de eventos posteriores, sino de relevancia, es decir, la verdad ha perdido relevancia en el tiempo.

Oxford Dictionaries (2017) cree que la composición de palabras pudo haber sido utilizado por primera vez en un 
ensayo de Steve Tesich, publicado por The Nation en 1992. Sin embargo, Ralph Keyes (2004), en su libro "The Post-Truth Era: Dishonesty and Deception in Contemporary Life", popularizó el término refiriéndose a él como la cualidad de que algo pareciera verdad, o pudiese percibirse como verdad, incluso si no lo es. A esta definición, añade que "un investigador tras otro ha confirmado que mentir se ha vuelto tan común como rascarse los puntos de una sutura" (Keyes, 2004 , p. 4) ${ }^{1}$. La post verdad se configura como afirmaciones, argumentos o eventos que pueden o no ser reales, no importa, lo relevante es que estos argumentos 0 eventos se sientan como verdaderos, aunque la evidencia muestre lo contrario.

La post verdad se hace y rehace de acuerdo a la necesidad, en tanto y cuanto la necesidad requiera su utilización para justificar usos culturales, razones individuales o, incluso, imposiciones estatales. Así, no es difícil encontrar ejemplos que representan con claridad estos usos culturales e individuales, imposiciones estatales o étnicas. Verbigracia, la mutilación genital femenina, pena de muerte por adulterio, felación realizada por niños, autoritarismo estatal, etc.

Sobre la mutilación genital femenina la OMS y la OPS $(2013$, p. 2) muestran datos que son escalofriantes ${ }^{2}$. Entre $100 \mathrm{y}$ 140 millones de niñas y mujeres en todo el mundo han sufrido de la mutilación genital femenina; en 27 países de África, y Yemen; más de 101 millones de niñas mayores de 10 años viven con las conse-

\footnotetext{
${ }^{1}$ Las traducciones al español de las citas en inglés han sido realizadas por el autor de este artículo.

${ }^{2}$ La palabra escalofriante ha sido utilizada con una intencionalidad específica: la palabra connota la existencia de una idea universal de bien y mal, de justo e injusto, bondad y crueldad, conceptos que no pueden justificar su existencia en la era de la post verdad.
}

cuencias de la mutilación; inmigrantes provenientes de África y Yemen siguen con estas prácticas en países como Australia, Canadá, Estados Unidos, Europa y Nueva Zelandia.

La mutilación genital femenina se realiza por varias razones culturales: iniciación de la edad adulta; entrar a la vida pública de la comunidad, esperando discriminación para aquellas mujeres que no se lo realizan; en diversas poblaciones, se ha vinculado esta práctica con tradiciones locales preislámicas; por razones que involucran creencias sanitarias y belleza; control de la sexualidad, "para frenar el supuesto deseo sexual intrínsecamente exacerbado de las mujeres (...) existe la creencia de que si el clítoris no es eliminado crecerá demasiado, pareciéndose a un pene" (Asociación Mujeres entre Mundos, 2016, pp. 21- 22).

Otro ejemplo de usos culturales se construye alrededor de la pena de muerte, uno de los casos más visibles, la muerte por adulterio, es común encontrarlo en ciertas culturas de oriente medio. Los debates sobre este uso son muy comunes en las diferentes comisiones de derechos humanos, de hecho, durante el debate sobre la pena de muerte en 1994, en el foro de las Naciones Unidas, los países que se opusieron a cualquier resolución sobre el tema fueron Malasia, Bangladesh, Sudan, Arabia Saudita, Libia, Egipto, Irán and Jordania. Sudán describió la pena capital como "un derecho divino según algunas religiones, en particular el islam (...)" (Schabas, 2000, p. 229). Cualquier intento de debate sobre tales prácticas recibe una denuncia feroz, aludiendo el respeto a los valores culturales.

Por otro lado, en Strickland-Bosav, ubicado en el norte de Papau Nueva Gui- 
nea, existen varios grupos indígenas, como por ejemplo los Kaluli quienes realizan varios rituales, uno de ellos recrea ambientes vecinos con canciones y danzas. Estos rituales traten de recordar memorias de seres perdidos, para ello, existen dos tipos de gentes, los danzantes y los anfitriones, quienes, "acongojados por el dolor, comienzan a llorar y llorar violentamente, hasta que algunos de ellos desahogan su dolor agarrando antorchas de resina ardientes y sumergiéndolas furiosamente en los hombros de la bailarina cantante" (Knauft, 1985, p. 324). El ritual se considera exitoso cuando los anfitriones se han perdido entre el llanto, la desesperación y los bailarines han terminado quemados severamente.

En la misma Strickland-Bosav, todos los grupos indígenas del área creen que los niños deben ser "inseminados para llegar a la edad adulta, pero el modo de transmisión del semen es diferente en cada sociedad" (Knauft, 1985, p. 328). Entre las comunidades Kaluli se practica el sexo anal, en la comunidad Etoro se practica la felación y en la comunidad Onabasulu se esparce el semen entre los novatos. Al mismo tiempo, a los Etoro les parece repulsivo las costumbres de los Kaluli y Onabasulu.

Por último, el ejemplo más visible del autoritarismo estatal es el de Corea del Norte, las dictaduras de la familia Kim y del Partido de los Trabajadores de Corea han creado un estado de represión sistemática. El régimen de Kim Jong-Un se ha caracterizado por las "ejecuciones públicas, detenciones arbitrarias y trabajos forzados; endurecer las restricciones de viaje para evitar que los norcoreanos escapen y busquen refugio en el extranjero; y sistemáticamente persiguiendo a aquellos con con- tactos religiosos dentro y fuera del país" (Human Rights Watch, 2017, p. 1). En el mismo reporte, se menciona el sistemático uso de asesinatos, esclavitud, tortura, encarcelamiento, violación, aborto forzado y actos de violencia sexual.

Las diferencias culturales conmueven y asustan a muchos, sin embargo, estas reacciones abren la puerta a preguntas relevantes: ¿Por qué debería asustarnos estos usos culturales, si la post verdad defiende la tolerancia cultural? ¿Por qué juzgar aquellos usos culturales, si no hay verdades absolutas, si no hay estándares absolutos? ¿Con qué derecho se pueden imponer estándares morales si éstos no son absolutos? Estas preguntas exponen el mayor problema de la argumentación en la era de la post verdad: no existe verdad, por ende, no existe principios morales, entonces, no hay justificación para juzgar a ninguna persona o cultura, en fin, no hay necesidad de leyes punitivas.

\section{Un "Cristo" de la post verdad}

En la era de la post verdad, muchas personas han intentado justificarse a través de la adaptación de un "Cristo" que tolere y no juzgue, que acepte y no niegue, un "Cristo" que se adapte a los cambios culturales. De la misma forma, se intenta construir una iglesia que se adapte, que tolere, que acepte, y se exige, simultáneamente, una comunidad que también se adapte a los diferentes estilos de vida. Los casos más claros de estos intentos de justificación y de presión para la adaptación son el homosexualismo y el aborto.

El caso del homosexualismo, como forma de vida aceptada en algunas iglesias cristianas en Gran Bretaña, ha sido 
estudiado por Hunt quien, citando a Robert Nugent y a Jeannine Gramick, menciona que "la homosexualidad se puede comparar con una espina atrapada en la garganta de la iglesia que no puede expulsar ni tragar por completo" (Hunt, 2002, p. 1). El estudio de Gramick muestra que la aceptación del homosexualismo en las iglesias cristianas se desenvuelve como un suceso sociológico, como el logro de la presión de algunos movimientos sociales. El homosexualismo "cristiano" como resultado de la presión social, y no por la aceptación de principios universales, o por la aceptación de la existencia de la verdad ${ }^{3}$.

La presión social y cultural revela el segundo problema en la argumentación en la era de la post verdad: la afirmación de que todo es una construcción social y, por lo tanto, como construcción social, la afirmación del más fuerte, del más adaptado, o de la mayoría, se convierte en el estándar moral, lo que involucra, nuevamente, la adaptación de los estándares. El problema de las mayorías es evidente: las mayorías son móviles y cambiantes, entonces, la consideración de algo verdadero depende de la construcción de las mayorías $y$, como éstas con móviles y cambiantes, lo verdadero también es móvil y cambiante.

Por otro lado, el aborto se ha convertido en la bandera de batalla de varios movimientos feministas, los cuales han basado sus argumentos en la libertad de las mujeres para decidir sobre sus cuerpos, sobre la salud y vida de la mujer, como también la discriminación de género (Center for Reproductive Rights, 2011).

\footnotetext{
3 Tanto el Antiguo Testamento (Levítico 18:22; 20:13), como el Nuevo Testamento (Romanos 1:2628; 1 Timoteo 1:9-10), se refieren al homosexualismo como actos contrarios a la voluntad de Dios para los seres humanos.
}

Estos argumentos han servido para crear ciertas justificaciones para aceptar legalmente el aborto. Sin embargo, en el caso de las estructuras de la legalidad internacional, Tozzi (2010, p. 6) menciona que ningún tratado de las Naciones Unidas contiene la palabra "aborto", ni puede inferirse un "derecho" al aborto del "sentido común" de las palabras de cualquier tratado.

La presión social ha hecho que grupos cristianos, como la Iglesia Presbiteriana de los Estados Unidos, acepte el aborto. En su declaración de principios menciona que:

"La decisión considerada de una mujer de interrumpir un embarazo puede ser una decisión moralmente aceptable, aunque ciertamente no la única ni la requerida. Las posibles circunstancias justificativas incluirían indicios médicos de deformidad física o mental grave, concepción como resultado de violación o incesto, o condiciones bajo las cuales la salud física o mental de una mujer o un niño estaría gravemente amenazada" (Central Presbyterian Church, 2013).

El mapa mundial sobre las leyes de aborto (Center for Reproductive Rights, 2018) muestra que el mundo está dividido en dos: un primer grupo conformado por aquellos países que han legalizado el aborto completamente, y un segundo que prohíbe el aborto de una u otra forma. En el segundo grupo se encuentra la mayor parte de América Latina, África y Asia Menor. Además, a estas regiones se agrega a Irlanda, Gran Bretaña, Polonia, Finlandia, Islandia, Nueva Zelanda, República de Corea y Japón. Estos países prohíben el aborto excepto en casos como incesto, violación o posible muerte de la madre, y 
en la mayoría se requiere el permiso del padre.

Los movimientos sociales a favor del aborto han pretendido construir la idea de legalidad sincronizada con la idea de moralidad. En esta concepción, se pretende sugerir que la legalidad del aborto muestra la moralidad de la nación. Sin embargo, este argumento deja entrever el tercer problema en la argumentación en la era de la post verdad: la legalidad no necesariamente representa la moral. Esta afirmación se presenta como una obviedad cuando se analiza el desarrollo histórico de los preceptos jurídicos de las naciones. El problema de la legalidad, entonces, enmarca la post verdad dentro de un desarrollo dinámico de los estándares legales, enmarca la post verdad dentro de una evolución histórica cambiante, una evolución que se la ve, a veces, como contradictoria, $y$, a veces, como coherente.

Estos dos ejemplos, la homosexualidad y el aborto, han servido como presión para construir una versión del cristianismo adaptado a la post verdad, que evoluciona de la misma forma que las sociedades, que camina los mismos senderos que las culturas de la post modernidad. Esta versión del cristianismo, adaptable al dinamismo cultural, se convierte en un engranaje más de la era de la post verdad porque sus preceptos son cambiantes, adaptables, sumisos a la cultura, sus principios caen en la subjetividad, en la opinión, en los gustos.

Los ejemplos también presentan la paradoja en la que caen ciertos movimientos sociales: la necesidad de ser justificado por la misma tendencia que acusa su comportamiento. Esta justificación muestra la necesidad de ser aceptado pero, al mismo tiempo, la voluntad de no cambiar sino, por el contrario, hacer que el cristianismo permute y se adapte a su conducta. Esta paradoja muestra la necesidad de una justificación espiritual, para ello, la opción más viable es la presión social para conseguir una fe cristiana adaptada.

\section{Los problemas de la post ver- dad}

El escrito ha presentado tres argumentos de la post verdad que recaen en problemas lógicos, a saber: el respeto cultural; la presión social; $y$, la legalidad. Todos estos problemas apuntan a un mismo argumento: la relatividad de la verdad, la relatividad de la moralidad y, por ende, la inexistencia de las dos. Por un lado, el respeto cultural conduce a aceptar cualquier uso cultural como violaciones, asesinatos, castraciones, etc. ¿Por qué espantarse, si la post verdad defiende la tolerancia cultural? El juicio a cualquier uso cultural cae en un absurdo, porque no hay verdades absolutas. Como no existe principios morales absolutos, la post verdad no puede justificar el juicio a ningún acto, el juicio a ningún ser humano.

Por otro lado, la presión social se construye como un instrumento que pretende construir la idea de moralidad a través de la imposición. Si todo es una construcción social, la verdad se construye en el contexto del más fuerte, aquel grupo de personas que logran imponer sus visiones sobre el resto. En muchos casos, las ideas de la mayoría se convierten en el estándar de moralidad. En otros casos, puede ser la fuerza de la minoría la que se imponga sobre una población. En ambos casos, sean mayorías o minorías, la imposición muestra la verdad como una idea 
relativa, cambiante. Así, la verdad y, por ende, la moralidad queda relegada a una pugna de fuerzas. La consideración de algo verdadero queda relegada a la construcción de las mayorías y, como éstas son móviles y cambiantes, la verdad no es absoluta, sino que depende del más fuerte.

Por último, la post verdad presenta la moralidad en relación con el sistema legal. La post verdad enmarca la moralidad dentro de la legalidad, las conductas son morales o no en tanto y cuanto se las pueda legalizar. De esta forma, la post verdad se desarrolla dentro de una evolución histórica de la legalidad, dentro de una evolución cambiante, dinámica, contradictoria. El problema con este argumento es obvio: la legalidad para unos es ilegalidad para otros, ¿Quién es moral y quién no? ¿En qué parámetros legales basamos nuestro juicio de moralidad? La dinámica de la legalidad muestra el mismo problema: una moralidad cambiante $y$, por ello, una moralidad inexistente.

Todos estos intentos de entender la moralidad desde la post verdad nos llevan a considerar el mismo problema: la dinámica de la sociedad ha construido parámetros históricos que son diversos, cambiantes y, por ende, inexistentes. Si la verdad se moviliza de un lado a otro, cambia sin problema, se adapta a cada cultura, entonces, la verdad ha desaparecido. Por lo tanto, no existe justificativo para entrar en juicios de valor, no existe un argumento razonable que justifique el juicio, la crítica, el modo de pensar o accionar. Solo existe la ley de imposición, de la persuasión, de la manipulación. En tanto y cuanto los grupos sociales puedan imponer, persuadir o manipular las concepciones culturales, la verdad sigue rele- gada al campo de la opinión, del placer, de lo subjetivo.

Como no existen parámetros objetivos para considerar que algo es verdad, para considerar que algo es moral, la post verdad se pierde en su intento de tolerancia. No puede existir tolerancia en el espectro de la post verdad por una razón evidente: si todo es tolerable, el razonamiento expuesto en este ensayo también debería ser tolerado, pero, si eso sucediera, si el razonamiento de este ensayo también es tolerado, la post verdad se contradice a sí misma porque la verdad y la no verdad no pueden coexistir en un mismo espacio. Por lo tanto, en el contexto de la post verdad, si los grupos sociales no toleran la argumentación de este artículo, se contradicen en su principio de tolerancia; por el contrario, en el contexto de la post verdad, si los grupos sociales sí toleran la argumentación de este artículo, se contradicen a sí mismos porque la verdad no puede coexistir con la no verdad.

Los argumentos esgrimidos en la era de la post verdad recaen en contradicciones lógicas. La única conclusión de esta era es la sistemática irracionalidad en la que se han construido sus "principios". Sin parámetros objetivos, el absurdo gobierna la razón. Sin parámetros objetivos, la cognición se pierde y aparece la incoherencia como elemento primario. Así, la post verdad vive de lo ininteligible, vive de lo subjetivo.

Sí lo expuesto tiene algún valor lógico, la pregunta siguiente es obvia, ¿Qué es la verdad? y ¿cómo llegar a ella? Estas preguntas nos llevan también a considerar la existencia de lo inmaterial, una verdad y una moralidad que no se construyen en la sociedad, no se construye en lo material, sino que se encuentra en el 


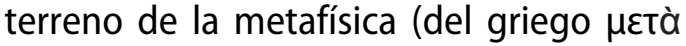

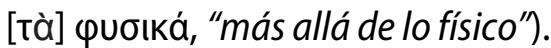

\section{4. ¿Qué/quién es la verdad?}

Si aceptamos que la verdad existe y es objetiva, entonces estamos aceptando que la verdad se encuentra por fuera de las construcciones culturales, se encuentra por fuera de las fuerzas materiales, se encuentra en el terreno de lo inmaterial. La verdad habita en el terreno de lo inmaterial porque no fue construida 0 creada por entes materiales, $y$, sin embargo, emerge en el terreno de lo material porque podemos apreciarla, aunque no la entendamos enteramente.

Los seres humanos no construimos la verdad porque ésta es independiente de los cambios y transformaciones que vivimos, es independiente de nuestras limitaciones. No obstante, sí la podemos entender porque hemos sido provistos de las leyes de la lógica, como la ley de no contradicción, la cual enfatiza su carácter de excluyente: la verdad y la post verdad no pueden coexistir. Para Tahko, la exclusividad de la ley de no contradicción es evidente:

"el énfasis está en la exclusividad mutua de tener un cierto atributo y carecer de ese atributo al mismo tiempo; de hecho, la idea puede expresarse sin usar la negación en absoluto: la exclusividad mutua de ciertas propiedades es evidente incluso sin el concepto de negación" (Tahko, 2009, p. 34).

La exclusividad es una característica ineludible de la verdad. Para ilustrar esta característica de la verdad, es muy útil referirnos a la parábola de los ciegos y el elefante. En esta parábola, un rey les pide a seis hombres ciegos que determinen qué están tocando al sentir diferentes partes del cuerpo de un elefante. Un ciego siente una pierna y dice que el elefante es como un pilar; el que siente la cola dice que el elefante es como una cuerda; el que siente el tronco dice que el elefante es como una rama de un árbol; el que siente el oído dice que el elefante es como un abanico; el que siente el vientre dice que el elefante es como una pared; y el que siente el colmillo dice que el elefante es como una pipa. Entonces el rey les explica: todos ustedes tienen razón.

La parábola de los ciegos y el elefante se utiliza para argumentar que todas las religiones son verdaderas, y que todas llegan al mismo Dios. Sin embargo, según Tim Keller, la parábola presenta varios problemas:

"Decir que no sé qué religión es verdadera es un acto de humildad. Decir que ninguna de las religiones tiene la verdad y que nadie puede estar seguro de que haya un dios, asume que tienes el tipo de conocimiento que simplemente dijiste que ninguna otra persona la tiene, que ninguna otra religión la tiene. ¡Cómo te atreves! Es una especie de arrogancia decir que nadie puede saber la verdad porque es una afirmación universal de la verdad. Decir: Nadie puede hacer afirmaciones universales de la verdad universales. Esa es una afirmación universal de la verdad. "Nadie puede ver toda la verdad", No podrías saber eso a menos que creas ver toda la verdad. $Y$, por 10 tanto, estás haciendo lo mismo que dices que las personas religiosas no deberían hacer" (Citado por Creitz, 2011).

Si los argumentos de la post verdad son ilógicos, porque no puede ha- 
ber múltiples verdades, sino solo una, como se ha intentado demostrar en los epígrafes anteriores, entonces, la verdad, exclusiva en su esencia, debe ser necesariamente lógica. Si la verdad es lógica, implícitamente aceptamos la existencia de una lógica inmutable, absoluta, una lógica que se encuentra fuera de tiempo y espacio. Entonces, como esta lógica no pudo haber sido creada en la materia, debe existir sin tiempo y espacio, debe ser un logos infinito, inmutable, absoluto.

El evangelio de Juan (1:1) nos ayuda a entender la naturaleza de este logos infinito, "En el principio ya existía el Verbo [^óyoc], y el Verbo [^óyoc] estaba con Dios, y el Verbo [^óyoc] era Dios". ^óyoc (logos) goza del principal atributo de la verdad: un logos que se encuentra por fuera de espacio y tiempo porque existe antes de la creación de la materia y, por ende, este logos es absoluto, inmutable. Este logos existe antes de la creación de la materia y estaba junto a Dios en una relación íntima. Este logos es Dios.

La verdad es lógica, y como los seres humanos hemos sido dotados de las leyes de la lógica, podemos apreciarla, aunque no podamos entenderla completamente. El logos es Dios, y puede ser apreciado por todo ser humano. En palabras de Anselmo (Davies y Leftow, 2004, p. 157):

1. Dios es algo que nada más grande puede ser pensado.

2. Dios existe en la mente ya que incluso el tonto puede pensar (tener en mente) en algo que nada más grande puede ser pensado.
3. Pero podemos pensar en algo que es más grande que algo que existe solo en la mente.

4. Entonces, algo que nada más grande puede ser pensado no puede solo existir en la mente.

De esta forma, la única posible vía de justificar la moralidad es a través de la verdad. Sin la verdad, la moralidad queda relegada a lo relativo. Es solo a través del logos que la moralidad puede tener sustento, puede tener justificación, la moralidad no puede existir fuera del logos. Así, la moral se ha convertido en un instrumento argumentativo útil para llegar a apreciar la existencia de un logos que se encuentra libre de las limitaciones del tiempo y espacio.

\section{Conclusiones}

Este ensayo ha pretendido mostrar los peligros de los postulados de la era de la post verdad, mientras se ha intentado relativizar lo relativo, de manera que se puede argumentar la irracionalidad de estos postulados. La irracionalidad de los postulados es evidente porque el respeto cultural, la presión social y la legalidad nos conducen al mismo punto: la imposible justificación de cualquier concepción de moralidad.

Los problemas de los postulados de la post verdad apuntan a la relatividad de la verdad y, por ende, la relatividad de la moralidad. La única forma de entender una estructura moral en la sociedad de la post verdad es a través de la imposición, de la persuasión o de la manipulación. En todos los casos, la moral queda relegada a lo subjetivo. 
Así, se ha propuesto que los argumentos de la post verdad son ilógicos, porque no puede haber múltiples verdades, sino solo una. Esta verdad es el logos, el cual se encuentra por fuera de espacio y tiempo porque existe antes de la creación de la materia. Este logos es Dios.

\section{Referencias Bibliográficas}

Asociación Mujeres entre Mundos. (2016). Guía para la conciencia sobre mutilaciones genitales femeninas. Andalucía: Junta de Andalucía Consejería de Igualdad y Políticas Sociales. Recuperado de https://bit.ly/2Fjw0u9

Center for Reproductive Rights. (2011). Safe and legal abortion is a woman's human right. Briefing paper. Recuperado de https://bit.ly/2tudUDa

Central Presbyterian Church. (2013). Our beliefs- abortion. Recuperado de https://bit.ly/2SKZXv7

Creitz, N. (20 de Octubre de 2011). The parable of the blind men and the elephant. Recuperado de https://bit.ly/2sAnWm7

Davies, B. y Leftow, B. (2004). The Cambridge Companion to Anselm. Cambridge: Press syndicate of the University of Cambridge. https://doi.org/10.1017/CCOL0521807468.

Human Rights Watch. (2017). North Korea country summary. Washington DC. Recuperado de https://bit.ly/2QkgHHH

Hunt, S. (2002). The lesbian and gay Christian movement in Britain. Journal of religion and society, 4, 1-19. Recuperado de https://bit.ly/2rUXlu8

Keyes, R. (2004). The post-truth era: dishonesty and deception in contemporary life. New York, NY: St. Martin Press.

Knauft, B. (1985). Ritual form and permutation in New Guinea: implications of symbolic process for socio-political evolution. American ethnologist, 12(2), 321-340. https://doi.org/10.1525/ae.1985.12.2.02a00080.

Koukl, G. (2017). The story of reality: how the world began, how it ends, and everything important that happens in between. Grand Rapids, Ml: Zondervan.

Koukl, G. (2011). Faith is not wishing: 13 essays for Christian thinkers. Signal Hill, CA: Stand to Reason.

Lennox, J. (2007). God's undertaker: has science buried God?. Oxford: Lios Hudson pcl.

Lennox, J. (2011). God and Stephen Hawking. Whose design is it anyway?. Oxford: Lion Hudson plc.

Lennox, J. (2015). Against the flow, the inspiration of Daniel in an age of relativism. Grand Rapids, Ml: Monarch Books. 
Organización Mundial de la Salud y Organización Panamericana de la Salud. (2013). Comprender y abordar la violencia contra las mujeres: mutilación genital femenina. Organización Mundial de la Salud. https://bit.ly/35mwc6v

Oxford University Press. (2016). Oxford word of the year 2016: Oxford languages. Recuperado de https://bit.ly/36osFpE

Schabas, W. (2000). Islam and the death penalty. William \& Mary Bill of rights journal, 9(1), 223-236. Recuperado de https://bit.ly/2tvQFZ5

Tahko, T. (2009). The law of non-contradiction as a metaphysical principle. Australian journal of logic, 7, 32-47. https://doi.org/10.26686/ajl.v7i0.1806.

Center for Reproductive Rights (2018). The world's abortion laws. Recuperado de https://bit.ly/2MQ1oEC

Tozzi, P. (2010). Internacional law and the right to abortion. International organizations law group legal studies series, (1). Recuperado de https://bit.ly/2Qk47Z2

Turek, F. (2014). Stealing from God: why atheists need god to make their case. Colorado Springs, CO: NavPress.

Turek, F. y Geisler, N. (2010). I don't have enough faith to be an atheist. Wheaton, IL: Crossway Books.

Zacharias, R. (2000). Jesus among other gods: the absolute claims of the christian message. Nashville, TN: Thomas Nelson Inc.

Zacharias, R. (2008). The end of reason. A response to the new atheists. Grand Rapids, Ml: Zndervan.

Zacharias, R. (2012). Why Jesus?. Rediscovering his truth in an age of mass marketed spirituality. New York, NY: FaithWords

\section{Para citar este artículo bajo Norma APA 6a ed.}

Naranjo Navas, C. (2019). De la post verdad a la verdad.

Cuadernos de Teología - Universidad Católica del Norte

(En línea), 11, e3088, https://doi.org/10.22199/issn.0719-

8175-2019-0008

Copyright del articulo: @2019 Christian Naranjo

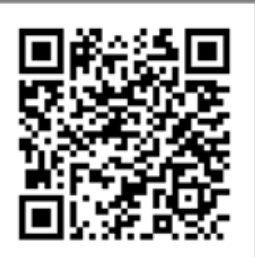

DOI

Este es un artículo de acceso abierto, bajo licencia Creative Commons BY 4.0 